\title{
SOBRE LA MIRADA DE GÉNERO EN LA SALUD REPRODUCTIVA Y LA CONSTRUCCIÓN SOCIAL DE LA MATERNIDAD
}

\author{
Esther Castellanos Torres y Isabel Soriano Villarroel \\ Femtopía Consulting \\ info@femtopia.es
}

Recibido: 28-02-2010

Aceptado: 22-04-2010

\section{Resumen}

El presente artículo contempla tres objetivos: 1) explicitar que la sexualidad engloba la reproducción humana; 2) reflexionar sobre la maternidad como construcción social y 3) entender la reproducción social como sustentadora del orden económico y social patriarcal. A partir de la lectura de fuentes secundarias de diferentes autoras que han abordado estas cuestiones desde un planteamiento constructivista de la maternidad y las relaciones de género, se ha reflexionado y estructurado el artículo en tres apartados que dan respuesta a los objetivos. Por último, se plantean conclusiones encaminadas a posibilitar el ejercicio pleno de los derechos sexuales y reproductivos.

Palabras clave: Derechos sexuales, Salud reproductiva, Maternidad, Reproducción social, Enfoque de género.

\footnotetext{
Abstract

This article seeks three objectives: 1) to clarify that sexuality includes also human reproduction, 2) to reflect on motherhood as a social construction and 3) to understand the social reproduction as a supporter of the patriarchal, social and economic order. The article answers the objectives in three sections from a point of view of a constructivist
} 
approach to the motherhood and the gender relations through the reading of secondary sources of several authoresses. Finally, the article sets some conclusions out in order to make possible the full exercise of sexual and reproductive rights.

Key words: Sexual Rights, reproductive health, motherhood, social reproduction, gender approach.

\section{Introducción y objetivos}

Como ya apuntó Silvia Caporale (2004), la capacidad de dar a luz es algo biológico, mientras que la necesidad de convertirlo en un papel primordial para las mujeres, es cultural. Esta diferencia que en principio resulta bastante obvia, en ocasiones se ha omitido por parte de instituciones, profesionales y mujeres, posicionando a estas últimas en situaciones de desigualdad respecto a los hombres.

Desde esta perspectiva, el presente artículo persigue dos grandes objetivos:

1. Explicitar que la sexualidad engloba dentro de sí misma a la reproducción humana.

2. Reflexionar sobre la maternidad como construcción social.

3. Entender la reproducción social como sustentadora del orden económico y social patriarcal.

Para dar respuesta a estas cuestiones se parte de dos premisas: por un lado, que la salud reproductiva forma parte integrante de la salud sexual, y por otro, que es necesario mirar la salud reproductiva desde el enfoque de género. Esto contribuirá a la ruptura de los mandatos tradicionales de género establecidos para las mujeres en la socialización diferencial, en tanto que seres reproductores-cuidadores, logrando un mayor empoderamiento de las mismas en sus elecciones y procesos de embarazo, parto y puerperio. 


\section{Material y métodos}

Para elaborar esta reflexión teórica acerca de cómo la maternidad es una construcción social ad hoc para la supervivencia del sistema patriarcal y capitalista, nos hemos nutrido del saber de distintas mujeres que han abordado estas cuestiones desde la rigurosidad científica y el pensamiento critico feminista. Se quiere destacar la tesis doctoral de Maribel Blázquez "Ideologías y prácticas de género en la atención sanitaria del embarazo, parto y puerperio: el caso del área 12 de la Comunidad de Madrid”, y el artículo de Lorena Saletti "Propuestas teóricas feministas en relación al concepto de maternidad", como documentos imprescindibles que han servido de base teórica y motivadora del presente artículo.

Además se ha realizado una revisión documental bibliográfica de fuentes secundarias como investigaciones, programas, informes y artículos, tomando aquellos que se sustentan en la teoría diferencial de género y excluyendo los de corte esencialista.

\section{La salud sexual como contenedora de la salud reproductiva}

La reproducción de mujeres y hombres es una parte más de la sexualidad. Esta afirmación se recoge en la Declaración del XIII Congreso Mundial de Sexología, 1997 Valencia (España), revisada y aprobada por la Asamblea General de la Asociación Mundial de Sexología (WAS) el 26 de agosto de 1999, en el XIV Congreso Mundial de Sexología (Hong Kong). En dicha Declaración se enuncian los 11 derechos sexuales que deben ser reconocidos, promovidos, respetados $\mathrm{y}$ defendidos por todas las sociedades y con todos los medios. El Derecho a tomar decisiones reproductivas libres y responsables, que encontramos en $8^{\circ}$ lugar, se refiere al "derecho a la toma de decisiones reproductivas libres y responsables. Esto abarca el derecho a decidir o no tener hijos, el número y el espacio entre cada uno, y el derecho al acceso pleno a los 
métodos de regulación de la fecundidad.” (Declaración del XIII Congreso Mundial de Sexología, 1997: 2)

Cabe recordar otras Conferencias Internacionales relacionadas con los derechos sexuales y reproductivos. En este sentido, un hito importante se produjo en 1994 cuando se celebra la Conferencia Internacional sobre la Población y el Desarrollo, en adelante CIPD, que supuso un gran cambio con respecto a las políticas de población que hasta entonces se habían discutido en foros internacionales. Hasta ese momento, el objetivo principal consistía en cumplir una serie de metas demográficas para frenar la superpoblación y paliar la pobreza. El interés en los temas reproductivos se debía por tanto a la importancia del control sobre la fecundidad como medio para alcanzar dichas metas demográficas (Mazarrasa y Gil, 2006).

En la CIPD surgen dos posturas contrapuestas, por un lado una perspectiva demográfica que aludía principalmente a las cuestiones de crecimiento, estructura y cambio de la población así como de sus repercusiones en el desarrollo social y económico, enfoque planteado también por gran parte del movimiento de planificación familiar. Por otro lado, la perspectiva que partía de la defensa de la salud y los derechos de las mujeres centrados en los desequilibrios de género y las necesidades sanitarias de las poblaciones.

La discusión entre esas dos posturas dio lugar al concepto de salud sexual y reproductiva, vinculando a nivel teórico el discurso sobre los derechos humanos y el derecho a la salud. Como resultado de las reivindicaciones del movimiento de mujeres, se produce un cambio de paradigma en el desarrollo humano consiguiendo que:

- Se rompa con la preocupación centrada únicamente en aspectos demográficos y de planificación familiar, asumiendo las necesidades en la salud de las personas, en especial de las mujeres, de un modo integral.

- Se fortalezca la reivindicación de los derechos sexuales y reproductivos por encima del interés por el control demográfico.

- Se defina la salud sexual a partir de la salud reproductiva. 
En esta Conferencia se introdujeron tres cambios fundamentales respecto a las conferencias anteriores (Ramos, 2006):

- El nuevo concepto de "salud reproductiva" es más amplio e integral que el de "planificación familiar".

- Se reconoce la sexualidad como una dimensión fundamental de las personas.

- Se considera que son las mujeres quienes deben controlar su propia fecundidad bajo una libre decisión, con condiciones que permitan que esto ocurra.

Aún así, el término "derechos sexuales" no logró ser incorporado en el documento final de consenso de la Conferencia del Programa de Acción de la CIPD, ya que resultaba bastante polémico llegar a un acuerdo en cuanto al término "derechos reproductivos" y aún más "derechos sexuales” (Ilkkaracan y Jolly, 2007). No obstante, el documento incluyó varios puntos importantes referentes a la sexualidad, entre ellos:

- Promover el desarrollo adecuado de una sexualidad responsable que permita el establecimiento de relaciones de equidad y respeto mutuo entre ambos sexos.

- Velar por que mujeres y hombres tengan acceso a la información, la educación y los servicios necesarios para lograr una buena salud sexual y ejercer sus derechos y responsabilidades en lo tocante a la procreación.

- Responsabilidad de los hombres en cuanto a su propia salud sexual y su fecundidad y los ayude a ejercer esas responsabilidades.

- Recomendaciones a los gobiernos y las comunidades que adopten medidas con carácter urgente para poner fin a la práctica de la mutilación genital de la mujer y proteger a las mujeres y las niñas contra todas las prácticas innecesarias y peligrosas de esa índole. 
- Se reconoce que la violencia sexual basada en género y los esfuerzos por controlar la sexualidad de las mujeres tienen impactos tanto en la salud de ellas como en su condición dentro de la sociedad.

- La sexualidad humana y las relaciones entre los sexos están estrechamente vinculadas e influyen conjuntamente en la capacidad del hombre y la mujer de lograr y mantener la salud sexual y regular su fecundidad.

Además en la CIPD, se recoge el concepto de una vida sexual satisfactoria:

"La salud reproductiva es un estado general de bienestar físico, mental y social, y no de mera ausencia de enfermedades o dolencias, en todos los aspectos relacionados con el sistema reproductivo y sus funciones y procesos. En consecuencia, la salud reproductiva entraña la capacidad de disfrutar de una vida sexual satisfactoria y sin riesgos y de procrear, y la libertad para decidir hacerlo o no hacerlo, cuando y con que frecuencia" (CIPD, 1994: Capítulo VII, Párrafo 7.2).

En el Programa de Acción de la CIPD se plantea que la salud reproductiva incluye también "la salud sexual, cuyo objetivo es el desarrollo de la vida y de las relaciones personales y no meramente el asesoramiento y la atención en materia de reproducción y de enfermedades de transmisión sexual" (CIPD, 1994: Capítulo VII, Párrafo 7.2).

Además, se alude explícitamente al papel que han de jugar los hombres en relación a la salud sexual y reproductiva:

"El hombre desempeña un papel clave en el logro de la igualdad de los sexos, puesto que en la mayoría de las sociedades, ejerce un poder preponderante en casi todas las esferas de la vida, que van de las decisiones personales respecto al tamaño de la familia, hasta las decisiones sobre política y programas 
públicos a todos los niveles. Es fundamental mejorar la comunicación entre hombres y mujeres en lo que respecta a las cuestiones relativas a la sexualidad y a la salud reproductiva, y la comprensión de sus responsabilidades conjuntas, de forma que unos y otras colaboren por igual en la vida pública y privada" (CIPD, 1994: Capítulo IV, Párrafo 4.24).

Después de El Cairo, en 1995 se celebra la IV Conferencia Mundial de la Mujer en Beijing. En ella, los derechos sexuales se convirtieron una vez más en un tema de gran debate. Hubo una gran resistencia al término "derechos sexuales" por parte de alianzas conservadoras, pero sin duda el asunto más controvertido fue la sexualidad, en especial la orientación sexual, el control de las mujeres sobre su propio cuerpo y el aborto (Ilkkaracan y Jolly, 2007).

Finalmente se aprobaron por unanimidad la Declaración y la Plataforma de Acción, constituyéndose como el parámetro a seguir por los gobiernos y la sociedad civil.

A modo de resumen general, la Plataforma afirma de nuevo que los derechos humanos de las mujeres incluyen su derecho a ejercer el control y decidir libre y responsablemente sobre las cuestiones relativas a su sexualidad, incluida su salud sexual y reproductiva, libres de coerción, discriminación y violencia y reconoce que la capacidad de las mujeres para controlar su fecundidad constituye una base fundamental para el disfrute de otros derechos. Además es indispensable para mejorar la salud de las mujeres, la responsabilidad compartida por la mujer y el hombre respecto de las cuestiones relativas al comportamiento sexual y reproductivo (Naciones Unidas, 1995).

Además, en el año 2003 el Parlamento Europeo y el Consejo de la Unión Europea publica el Reglamento 1567/2003 del Parlamento Europeo y del Consejo relativo a la ayuda para políticas y acciones sobre la salud y derechos en materia de reproducción y sexualidad en los países en desarrollo, en el Artículo 1.2 se apuesta por: "un enfoque holístico y el reconocimiento de la salud y de los derechos en materia de reproducción y sexualidad [...], incluida una maternidad exenta de riesgos y el acceso 
universal a una gama completa de cuidados y servicios seguros y fiables en materia de salud reproductiva y sexual".

Dicho esto, la causa de que el abordaje de la salud sexual no se haya desarrollado desde una perspectiva holística, centrándose de manera exclusiva en la salud reproductiva, se debe principalmente a las altas tasas de mortalidad materno infantil que han acompañado a las mujeres a lo largo de la historia, y que todavía hoy, en numerosos países, siguen siendo una realidad. Es por esto que los movimientos feministas y de mujeres pedían y piden a través de la agenda política de las diferentes Conferencias Internacionales, actuaciones específicas en torno a la salud reproductiva, en concreto a la atención médica durante el embarazo, parto y puerperio.

Sin duda, esta necesidad de primer orden, hace que la mayor parte de los esfuerzos realizados por los gobiernos en materia de salud sexual y reproductiva vayan dirigidos a la atención al embarazo, parto y puerperio, con diferencias dependiendo de los contextos socioeconómicos y culturales de los países. Este hecho ha traído consigo dos cuestiones: la invisibilización de la sexualidad entendida de manera integral por un lado, y la omisión del enfoque de género en el hacer biomédico de la salud reproductiva por otro.

Por todo ello, es necesario atender la sexualidad y la salud sexual desde una mirada de género, lo cual significa poner el foco en los derechos sexuales y reproductivos.

\section{La construcción social de la maternidad}

En todas las sociedades históricas conocidas, la maternidad ha sido considerada la condición femenina por excelencia, entendiéndola en ocasiones incluso como la misma esencia femenina. Sin duda, como apunta Lorena Saletti (2008), ha sido la capacidad biológica de procrear de las mujeres la que ha sustentado la permanente identificación entre feminidad y maternidad. 
Por su parte, autoras como Nancy Chodorow (1984), señalaron el ejercicio maternal de las mujeres como el punto central de la división sexual del trabajo, promoviendo la necesidad de estudiar la noción de «maternaje» como el resultado de un proceso cultural, que ha asignado históricamente a las mujeres el papel de cuidadoras. De esta manera, el ejercicio maternal de las mujeres determina su localización en la esfera doméstica de la sociedad, reproduciendo la identidad genérica que se adquiere a través de la socialización, uno de los pilares del mantenimiento de la división sexual del trabajo y de las relaciones de poder entre mujeres y hombres.

De la misma manera, Simone De Beauvoir indicó ya en 1949 que la condición social femenina no es sólo un efecto de la diferencia sexual, sino sobre todo una consecuencia de la socialización de las mujeres que tiene lugar en todos los ámbitos de su vida cotidiana, entre los que destaca de nuevo la maternidad.

De esta manera se explica cómo tradicionalmente, y con los cambios en la industrialización, la sociedad ha asignado a hombres y mujeres diferentes roles y tareas estereotipadas a través de la división sexual del trabajo, siendo para unos fecundar, proveer el sustento, ejercer la autoridad y defender a la familia, y para otras ocuparse del cuidado del hogar, la maternidad, velar por la salud de quienes integran la familia y brindar afecto. Estos dos grupos de tareas se diferencian claramente por el lugar en el que se realizan, configurando la división en los usos del tiempo y de los espacios domésticos para las mujeres, y los espacios públicos para los hombres (aunque cada vez más también para las mujeres), estableciendo por tanto un contrato social, laboral y de género. Así pues, las mujeres contemporáneas ocupan cada vez más espacios públicos desarrollando actividades productivas remuneradas, mientras siguen asumiendo las tareas domésticas, sin que haya una reciprocidad de funciones por parte de los hombres.

Esta realidad actual no es sino el continuo de la historia de las mujeres, en quienes ha recaído la responsabilidad de la maternidad y la crianza, así como el cuidado de las personas dependientes. Es decir, las mujeres han incorporado por mandato de género el cuidado de otras personas, independientemente de nuestra posible condición de madres, por lo que las mujeres que han optado por la no reproducción, o que 
simplemente no han tenido oportunidad de ejercerla, no se libran de las tareas vinculadas con la reproducción social. Esto, aunque el presente artículo se centre únicamente en cuestiones relacionadas con la maternidad, es muy importante no perderlo de vista.

Otra de las cuestiones estructurales que ha hecho posible esta división sexual del trabajo, tiene que ver con que ni a las políticas públicas, ni a las parejas, les ha interesado hacer de la procreación y la crianza un acto más allá de lo meramente biológico. De haberlo hecho, tendrían que haber puesto los medios necesarios para contribuir a su desarrollo, tambaleando los cimientos del sistema patriarcal y capitalista de dominación. Por tanto, los Estados omiten su responsabilidad de crear recursos públicos para hacerse cargo de los cuidados a personas dependientes como escuelas infantiles, centros de día o residencias, mientras que los hombres se despreocupan del uso de métodos de prevención de ITS y anticonceptivos, así como de la reproducción social vinculada en este caso al cuidado y la crianza de las criaturas.

Como ya se ha dicho, Saletti (2008) insiste en que la posibilidad biológica de dar a luz se convierte en un mandato social para las mujeres a través de la afirmación del instinto materno universal. Dicha afirmación garantizará que se asuma -y que las propias mujeres asuman- que tienen la obligación de ser madres. Gracias a este supuesto instinto, la maternidad biológica se transforma en maternidad sociológica, en hecho social, logrando que las diferencias biológicas entre los sexos se conviertan en la base del sometimiento de las mujeres. El mito del instinto maternal, supuestamente natural e intrínseco, predestina a las mujeres a ser madres para posteriormente dedicarse con prioridad al cuidado de las criaturas que dan a luz. Es necesario resaltar que no se encuentra ningún correlato equivalente en el caso de los hombres.

La maternidad, y en especial su ejercicio, condicionan la identidad de género relacional, típicamente femenina (Hernando, 2008). El mito del instinto maternal es un claro exponente de la utilización de datos biológicos (como el útero, el embarazo o el parto) con fines de opresión y aislamiento de las mujeres en las funciones reproductivas. 
De esta forma, la construcción de la maternidad se fundamenta en la relación natural y biológica de la madre con su hijo o hija, en el presunto instinto maternal y en la necesidad del "vínculo materno-filial", mientras que la paternidad se construye como una función social elaborada por la cultura (Blázquez, 2009). El proceso de construcción de la maternidad supone la generación de una serie de mandatos relativos a su ejercicio, normas que se encarnan en las personas e instituciones y que son reproducidas en discursos, imágenes y representaciones de todo tipo, produciendo de esta forma un ideal maternal (Saletti, 2008).

Todo este conjunto de naturalizaciones -en el sentido de que son afirmaciones que se han ido estableciendo, percibidas actualmente de una forma natural, ahistórica y asocial- siguen vigentes, aunque bajo formas tan complejas como nuevas. Así, los cambios legales y sociales que han permitido el acceso a la educación, a los derechos sexuales y reproductivos, al trabajo remunerado y a la autonomía para las mujeres, como también las nuevas formas de familia y de convivencia, han contribuido por ejemplo a que se tengan muchos menos hijos e hijas, focalizando todo el cuidado y la atención en ellos y ellas (Blázquez, 2009).

Por tanto, la ideología de la maternidad (Albite-Vélez \& Valle-Ferrer, 2003; Golden, 1998) se construyó bajo el reclamo del sacrifico y abnegación femenina en pro del bienestar familiar, sentando las bases para la opresión femenina en el ámbito doméstico, privado y público, así como para las múltiples formas de violencia contra las mujeres dentro y fuera del hogar (Rodríguez, 2008). En este sentido, entre las manifestaciones de las violencias ejercidas contra las mujeres en relación sexualidad y reproducción, cabe destacar la violencia sexual, los abortos inducidos o provocados, la mutilación genital femenina y los embarazos forzosos. Respecto a esto último, los derechos sexuales y reproductivos presuponen que las hijas e hijos son decididos, pues existen medios anticonceptivos para su planificación, aunque lo cierto es que existen embarazos llevados a término que no son deseados.

Numerosas autoras por tanto afirman que la maternidad no es un hecho natural, y que el proceso de procreación humana está mediado por factores culturales que 
determinan quienes están en condiciones de hacerlo y quiénes no, de qué forma hacerlo y con quienes. Incluso, en qué momento de sus vidas llevarlo acabo, según clase social, pertenencia étnica, religiosa, época histórica y ubicación geográfica (Knibiehler, 2001; Navarro, 2002; Arango, 2001; Di Marco, 1997; Tubert, 1993).

Tal y como afirma Maribel Blázquez (2009), la naturalización de los procesos femeninos conduce por tanto a una visión esencialista y determinista de las mujeres, excluyendo otros factores que pudieran estar interviniendo en su toma de decisiones. También impide pensar en las "rupturas" que pueden producirse en función de los entornos sociohistóricos en que se desarrolla la vida de las mujeres o las condiciones sociohistóricas en las que la medicina elabora sus discursos y conforma sus prácticas. Se excluyen así también las diferencias entre las mujeres en el deseo del embarazo, en sus formas de sentir y afrontar el dolor, en la capacidad de resistir al mismo, de cuidar su vida y la vida de su criatura, de percibir y vivir el amamantamiento, etc. Estas interpretaciones están ordenadas, además, dentro de unas concretas relaciones sociales de género, donde las mujeres son vistas principalmente como madres y cuidadoras.

Como consecuencia tanto del determinismo biológico como de la naturalización, se considera que todas las mujeres son iguales, tienen una biología común y su biología se comporta igual en todas ellas (Op. cit.). Es necesario considerar las biografías, contextos y circunstancias en los procesos de salud y enfermedad de cada mujer, ya que por ejemplo, todas tienen el mismo deseo de un buen parto, pero no todas parten de las mismas condiciones para tener a sus criaturas de una forma saludable (Lazarus, 1988).

A pesar de esto, la maternidad es uno de los ámbitos de la salud y de la experiencia general donde los factores sociales, de género y culturales son más invisibles, tanto para la población general como para profesionales de la salud (Esteban, 2000).

Así, desde la asistencia sanitaria se refuerza el ideal maternal esencialista desde el mismo momento del embarazo, cuando las y los profesionales nombran a las mujeres como madres antes de serlo, elaborando a través de los discursos la centralidad de la maternidad en la vida de las mujeres y la creación de los vínculos con la futura criatura. 
Por ejemplo, se advierte a las mujeres que optan por la lactancia artificial o plantean que sus parejas disfruten de la baja maternal, todos los inconvenientes que conllevan estas elecciones. Lo cual no sucede al contrario, es decir, a las mujeres que quieren lactancia materna y disfrutar de la baja de maternidad no se les explican los inconvenientes que esto supone para ellas, para sus criaturas y para sus parejas (Op. cit.).

Bajo esta óptica, se invisibiliza que las relaciones y vínculos entre las mujeres y sus criaturas son construidas, siendo en realidad el resultado de una experiencia entre la criatura cuidada y las personas que la cuidan. De esta manera, en el caso de cada mujer puede ir surgiendo o no el vínculo en la medida en que avanza su embarazo, se sucede el parto, se recupera del mismo, va relacionándose con su criatura y descubre habilidades propias para el manejo de las distintas situaciones. Entre los factores que se nombran para el no establecimiento del "buen vínculo", se encuentran la falta de información de las mujeres sobre estas cuestiones, el que no hayan tenido "partos fisiológicos", la incorporación al trabajo remunerado, la brevedad de la baja maternal y la deshumanización de la atención (Blázquez, 2009).

Por ello, es importante recoger lo que apunta Maribel Blázquez (2009), que la deconstrucción de la maternidad como hecho natural, teniendo en cuenta las distintas experiencias entorno a este proceso que viven mujeres y hombres, dependen de la cultura, de lo aprendido socialmente y no de la "naturaleza" y de lo "innato".

\section{La maternidad y la reproducción social}

Es necesario entender la forma en que las relaciones de género sustentan el orden económico-social e inciden en su evolución y, a la vez, cómo los cambios en este orden afectan el sistema de género. Ya se ha visto como el rol maternal tiene efectos profundos en la vida y la identidad de las mujeres, en lo que se espera de ellas, en la reproducción también de la masculinidad tradicional, en la desigualdad entre los sexos y en la generación de formas precisas de poder laboral. 
Tal y como han mostrado numerosas escritoras, las alianzas establecidas entre el patriarcado y el capitalismo son absolutamente necesarias para mantenerse como sistemas dominantes. Su sustento se basa, en primer lugar, en las actividades que las mujeres realizan en sus casas y que vienen a dominarse "tareas domésticas" o "reproducción social", concepto que se utiliza el este artículo.

Se entiende por reproducción social el proceso dinámico de cambio vinculado a la perpetuación de los sistemas sociales, que involucra tanto factores económicos como ideológicos, políticos y sociales en un proceso de mutua influencia. Se refiere también a la reproducción de las condiciones que sostienen un sistema social: la cuestión fundamental es qué estructuras se tienen que reproducir para que pueda reproducirse la sociedad en su conjunto. Implica la transmisión del acceso y el control de recursos económicos de una generación a otra, lo cual varía con el tipo de organización social que en este caso es patriarcal y capitalista. Cabe señalar por tanto que hay una correspondencia entre la organización de trabajo productivo y el tipo de organización del trabajo reproductivo que le sirve de sustento, que marca y a la vez muestra un sistema de relaciones de género (Todaro y Yáñez, 2004).

Históricamente, tal y como apunta Cristina Segura (2004), las tareas vinculadas con la reproducción social no reciben retribución alguna, no devengan derechos y no tienen valor social. La crianza de las criaturas por parte de las mujeres es una de estas tareas dirigidas al bienestar y al mantenimiento de las personas que forman la unidad familiar. Estas tareas no han sido reconocidas como imprescindibles para el desarrollo de la vida ni validadas como actividades económicas, es más, desde los diferentes modelos de producción se ha hecho creer que la reproducción social no produce riqueza, justificando así su falta de retribución económica.

Hay que poner de manifiesto una vez más como la reproducción social supone un gran ahorro para el sistema de producción capitalista, ya que se utiliza a las mujeres como mano de obra gratuita y se exime a los Estados de poner los recursos públicos necesarios para proveer las necesidades de cuidados de sociedades cada vez más envejecidas. La familia patriarcal se define, por tanto, como un espacio que genera 
plusvalías, derivadas de todos los trabajos que las mujeres llevan a cabo sin recibir ningún tipo de remuneración, pero de los que se benefician los hombres, y sobre todo el Estado, gracias como se ha dicho a la mano de obra gratuita que aportan las mujeres.

Es más, Anderson (1991: 24) señala que "el trabajo doméstico, olvidado en la caja negra de la reproducción social, no figura en las cuentas nacionales, ni incide en el cálculo de la productividad o la riqueza de país alguno, ni (aparentemente) consume el tiempo o las energías de quienes lo realizan". En este sentido, se ha valorado la repercusión que tendría en el PIB si el Estado supliera la mano de obra gratuita de las mujeres atendiendo a criaturas, personas enfermas, ancianas y discapacitadas de las familias. La conclusión a la que se ha llegado es que, independientemente del sistema de producción, en cualquier país la economía entraría en una profunda crisis (Segura, 2004).

Estas reflexiones en torno a la entera disponibilidad de las mujeres para la crianza de las criaturas no pueden obviarse a la hora de teorizar o actuar sobre la salud reproductiva y la maternidad. Omitir estas variables supone mantener las relaciones de poder-subordinación, encadenando a las mujeres a las tareas domésticas, a la vez que van asumiendo cada vez más trabajos productivos remunerados.

\section{Conclusiones y recomendaciones}

En primer lugar, en la misma línea que Alice Miller (2000), consideramos que el papel de los Estados es crear las condiciones necesarias para que las mujeres y los hombres ejerzan sus derechos sexuales respecto a la elección de si enlazar o no la sexualidad con la reproducción. Esta idea debería permitir, en el marco de las demandas sobre los derechos sexuales, atender con igualdad a una más amplia gama de identidades, prácticas y condiciones sexuales, que actualmente no pueden ser protegidas al tratar a los derechos sexuales como un subconjunto de los derechos reproductivos.

En segundo lugar, y aterrizando al caso de España, cabe destacar como los avances en las políticas públicas en el ámbito de la salud como la Estrategia de 
Atención al Parto Normal en el Sistema Nacional de Salud del año 2007, han supuesto la satisfacción de las necesidades prácticas de las mujeres mejorando los servicios de salud materno infantiles, la salud perinatal, la atención al parto normal, etc. Aun así, ahora es el momento de dar un paso más y acompañarlas con políticas de transformación de las relaciones de género, que garanticen el ejercicio pleno de los derechos sexuales y reproductivos de las mujeres y los hombres.

Tener en cuenta las necesidades estratégicas de género en las políticas públicas, contribuirá a revertir las inequidades construidas históricamente en la sexualidad y la reproducción, así como sus efectos concretos en la vida de mujeres y hombres. En este sentido, tendremos que estar atentas al desarrollo de la Ley Orgánica 2/2010, de 3 de marzo, de salud sexual y reproductiva y de la interrupción voluntaria del embarazo en sus diferentes ámbitos relacionados con la educación sexual a población y profesionales, con las iniciativas de estrategias y programas de salud sexual y reproductiva, con la financiación de los métodos anticonceptivos e IVE`s, y con su práctica en los servicios sanitarios públicos.

En tercer lugar, para contribuir al empoderamiento de las mujeres en su sexualidad, es necesario revisar el concepto del "rol reproductivo" de las mujeres introduciendo su derecho a una salud sexual plena. Las mujeres, además de madres, son también sujetos con derechos (derecho a la sexualidad sin riesgos, derecho a decidir sobre el propio cuerpo y la maternidad, derecho a elegir pareja, derecho a elegir el número de hijos e hijas que se desea tener, etc.), por lo que la implicación de las parejas es fundamental para sostener este proceso de cambio, ya que deben considerarse coresponsables en el cuidado tanto de las propias mujeres como de las criaturas.

Mirar la salud reproductiva desde el enfoque de género, como apunta Maribel Blázquez (2009), supone reconocer que el papel de la pareja ha estado descuidado por los Estados en general, y por los sistemas sanitarios en particular. Para generar cambios estructurales en las relaciones de género es necesario su participación en el desarrollo de los derechos y responsabilidades que acarrea la llegada de una nueva criatura a la unidad familiar, por lo que desde un planteamiento de equidad, deben distribuirse las 
responsabilidades y deberes referidos a la sexualidad y a la reproducción entre ambas partes de la pareja.

Se puede añadir incluso que como apunta Alejandra López y su equipo (2003), mirar la salud sexual desde la perspectiva de género significa entender la salud reproductiva como un proceso multidimensional, que integra los aspectos vinculados con el ciclo vital de las mujeres y hombres, así como el ejercicio pleno de sus derechos sexuales y reproductivos en tanto derechos humanos. Esta mirada permitirá complejizar el diseño e implementación de las políticas de salud ajustándolas a las necesidades prácticas y estratégicas de las mujeres.

En cuarto lugar no hay que olvidar que existe la posibilidad de que las mujeres puedan afrontar la maternidad sin pareja. Puesto que esta realidad existe, desde los servicios de salud se deben poner las medidas necesarias para no discriminar a estas mujeres, garantizándolas el ejercicio de sus derechos de la misma manera que al resto de mujeres.

Finalmente, teniendo en cuenta lo apuntando anteriormente respecto a la reproducción social, es preciso señalar que el contrato social que se desarrolló a partir de las relaciones existentes en el régimen industrial de producción, y que se ha visto erosionado con el advenimiento de la era informática y los cambios socioculturales, debe ser reemplazado por un nuevo contrato laboral y de género, que dé espacio al desarrollo normal de la fuerza de trabajo actual y futura según las nuevas condiciones económicas, sociales y culturales. 


\section{BIBLIOGRAFÍA}

- Albite-Vélez, L. y Valle-Ferrer, D. (2003): "La ideología de la maternidad en la subjetividad femenina: Mecanismo de opresión y violencia doméstica”. En L. Martínez Ramos y M. Tamargo López (eds.): Género, sociedad y cultura). San Juan, Puerto Rico: Publicaciones Gaviota, pp. 110-134.

- Anderson, J. (1991). Reproducción social / Políticas sociales. Lima: SUMBI.

- Arango, G.; León, M. y Viveros, M. (comp.) (2001): Género e identidad. Ensayos sobre lo femenino y lo masculino. Madrid: TM.

- Asociación Mundial de Sexología WAS (1999): "Declaración del 13avo. Congreso Mundial de Sexología, 1997, Valencia, España”. Revisada y aprobada por la Asamblea General de la Asociación Mundial de Sexología, WAS, el 26 de agosto de 1999. En $14^{\circ}$ Congreso Mundial de Sexología. Hong Kong, República Popular China, [en línea] Disponible en: http://www.redeser.org/decladerecsexu.pdf [02/01/2010].

- Blázquez Rodríguez, Mª I. (2009): Ideologías y prácticas de género en la atención sanitaria del embarazo, parto y puerperio: el caso del área 12 de la Comunidad de Madrid. Universitat Rovira I Virgili.

- Caporale Bizzini, S. (coord.) (2004): Discursos teóricos en torno a la(s) maternidad(es): una visión integradora. Madrid: Etinema.

- Chodorow, N. (1984): El ejercicio de la maternidad. Psicoanálisis y sociología de la maternidad y paternidad en la crianza de los hijos. Barcelona: Gedisa.

- De Beauvoir, S. (1949). El segundo sexo. Vol. I y II. Madrid: Cátedra Feminismos.

- Di Marco, G. y Schmuckler, B. (1997): Madres y democratización de la familia en la Argentina contemporánea. Buenos Aires: Biblos.

- Esteban, M'. L. (1993): "La salud de las mujeres: nuevas preguntas para nuevas respuestas”. En M. C. Díez y V. Maquieira (eds): Sistemas de género y construcción (deconstrucción) de la desigualdad. Granada. Actas del VI Congreso de Antropología. 
. (2000). "La maternidad como cultura: algunas cuestiones sobre lactancia materna y cuidado infantil". En Perdiguero, Esteban; Comelles, E. (eds.): Medicina y cultura. Estudios entre la antropología y la medina. Barcelona, pp. 207-226.

- Guía para programas y proyectos de salud sexual y reproductiva en África (2005): Programa VITA, Programa de Cooperación al Desarrollo en Salud para África y Agencia Española de Cooperación Internacional (AECI).

- Golden, S. (1998): Slaying the mermaid: Women's culture of sacrifice. New York: Harmony Books.

- Hernando, A. (2008): “Género y sexo. Mujeres identidad y modernidad”. En Claves de la Razón Práctica, nº 188, pp. 64-70.

- Ilkkaracan, P. y Jolly, S. (2007): Género y sexualidad. Informe General Brighton: Bridge Development Gender.

- Knibiehler, Y. (2001): Historia de las madres y de la maternidad en Occidente. Buenos Aires: Nueva Visión.

- Lazarus, E. (1988): "Poor women, poor outcomes: social class and reproductive health". En Michaelson K.: Childbirth in America: Anthropology perspectives. South Hadley Mass: Bergin and Garvey, pp. 39-54.

- López Gómez, A.; Benia, W. y Contera, M.; Güida, C. (2003): Del enfoque materno infantil al enfoque de la salud reproductiva. Tensiones, obstáculos y perspectiva. Montevideo, Uruguay.

- Mazarrasa, L y Gil, S. (2006): Módulo 12: Salud Sexual y Reproductiva, dentro del Programa de Formación de Formadores/as en Perspectiva de Género y Salud del Observatorio de Salud de las Mujeres (Ministerio de Sanidad y Consumo y la Universidad Complutense de Madrid).

- Miller, A. (2000): "Sexual not reproductive: exploring the junction and disjunction of sexual and reproductive rights". En Health and Human Rights, vol. 4, no. 2, Harvard College.

- Naciones Unidas (1995): “Cuarta Conferencia Mundial sobre la Mujer”. Beijing, [en línea] Disponible en: http://www.acnur.org/biblioteca/pdf/4654.pdf [02/01/2010].

- CIPD - Naciones Unidas (1994): "Programa de Acción de la Conferencia Internacional sobre Población y Desarrollo", El Cairo, [en línea] Disponible en: 
http://www.cinu.org.mx/temas/desarrollo/dessocial/poblacion/icpd1994.htm\#i1 [02/01/2010].

- Navarro, M. y Stimpson C. (comp.) (2002): Sexualidad, género y roles sexuales. Madrid: Fondo de Cultura Económica.

- Ramos Padilla, M. (2006): "La salud sexual y la salud reproductiva desde la perspectiva de género". En Revista Peruana de Medicina Experimental y Salud Pública, jul-sep, vol. 23, no. 3, pp. 201-220.

- Reglamento (CE) n ${ }^{\circ}$ 1567/2003 del Parlamento Europeo y del Consejo, de 15 de julio de 2003, relativo a la ayuda para políticas y acciones sobre la salud y derechos en materia de reproducción y sexualidad en los países en desarrollo.

- Rodríguez, V. (2008): "Reflexiones sobre el Género, la Maternidad y el Éxito profesional de las mujeres”. En Revista Kálathos.

- Saletti, L. (2008): "Propuestas teóricas feministas en relación al concepto de maternidad”. En Clepsydra, pp. 169-183.

- Segura, C. (2004): "Mujeres, trabajo y familia en las sociedades preindustriales". En La Historia de las mujeres una revisión historiográfica. Universidad de Valladolid, pp. 229-248.

- Todaro, R. y Yáñez, S. (2004): El trabajo se transforma: relaciones de producción y relaciones de género. Centro de Estudios de la Mujer. Chile.

- Tubert, S. (1993): La construcción de la feminidad y el deseo de ser madre, cuerpo y subjetividad femenina, salud y género. Madrid: Siglo XXI. 